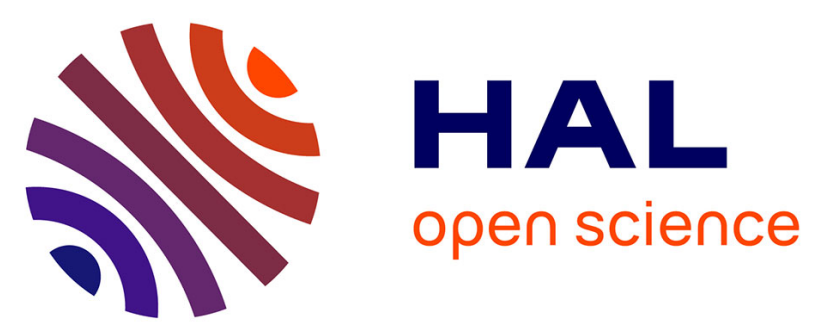

\title{
Standardization of a Multiplex Magnetic Bead-based for Simultaneous Detection of IgG to Plasmodium Antigens
}

Ronald Perraut, M-L Varela, Babacar Mbengue, M. Guillotte, Odile Mercereau-Puijalon, Inès Vigan-womas

\section{To cite this version:}

Ronald Perraut, M-L Varela, Babacar Mbengue, M. Guillotte, Odile Mercereau-Puijalon, et al.. Standardization of a Multiplex Magnetic Bead-based for Simultaneous Detection of IgG to Plasmodium Antigens. Journal of Immunological Techniques \& Infectious Diseases, 2015, 4 (2), pp.1-8. 10.4172/2329-9541.1000134 . hal-01299922

\section{HAL Id: hal-01299922 \\ https://hal.science/hal-01299922}

Submitted on 8 Apr 2016

HAL is a multi-disciplinary open access archive for the deposit and dissemination of scientific research documents, whether they are published or not. The documents may come from teaching and research institutions in France or abroad, or from public or private research centers.
L'archive ouverte pluridisciplinaire HAL, est destinée au dépôt et à la diffusion de documents scientifiques de niveau recherche, publiés ou non, émanant des établissements d'enseignement et de recherche français ou étrangers, des laboratoires publics ou privés. 
Standardization of a Multiplex

Magnetic Bead-based for

Simultaneous Detection of IgG to Plasmodium Antigens

\section{Perraut $\mathbf{R}^{1}$, Varela $\mathbf{M}-\mathrm{L}^{1}$, Mbengue $\mathbf{B}^{2}$, Guillotte $\mathbf{M}^{3}$, Mercereau-P uijalon $\mathrm{O}^{3}$ and Vigan-Womas $\mathrm{I}^{3}$ \\ ${ }^{1}$ Institut Pasteur de Dakar, Unité d'Immunologie, Dakar, Sénégal \\ ${ }^{2}$ Institut Pasteur de Dakar, Unité d'Immuno-Génétique, Dakar, Sénégal}

${ }^{3}$ Institut Pasteur, Unité d'Immunologie Moléculaire du Parasite, 25-28 Rue du Dr Roux, 75015 Paris, France

"Corresponding author: Ronald Perraut, Institut Pasteur de Dakar, Unité d'Immunologie, Dakar, Sénégal, E-mail: perraut@pasteur.sn

Rec date: Oct 01, 2015 Acc date: 08, 2015 Dec Pub date: Dec 14, 2105

\section{Abstract}

Background: Multiplex assays are currently used to facilitate the evaluation of antibody $(\mathrm{Ab})$ responses to multiple Plasmodium falciparum antigens from large field-based epidemiological studies. The present study aimed at (i) optimizing parameters of a novel cost-effective, compact and reliable magnetic bead-based multiplex immunoassay (MBA) carried out with the MAGPIX ${ }^{\circledR}$-Luminex system and (ii) comparing the results with those obtained using standard ELISA technology.

Methods: Several MBA parameters including antigen amount for coupling, plasma dilution, type of plates, buffers, washing procedure to minimize bead loss, and bead quantity for testing were optimized. Antibody responses to two recombinant and two peptidic $P$. falciparum antigens, one Anopheles gambiae salivary gland peptide gSG6 and Bovine Serum Albumin as negative control were tested by MBA and ELISA using sera from 14 villagers from an hyper-endemic Senegalese village.

Results: The MBA procedures were developed to reflect responses observed in the standard ELISA protocols used in previous studies. Using the finalized MBA protocol, a strong significant positive correlation $(P<10-3)$ was observed between ELISA and multiplex-MFI (median fluorescence intensity) antibody readouts (PfMSP1p19, PF13-DBL1a1 recombinant proteins, rho $=.77$ and .82 , respectively; LSA141, CSP and gSG6, synthetic peptides; rho $=.86, .61$ and .73 , respectively). Backgrounds with the negative control BSA or non-immune sera were minimal. There was a good reproducibility of MFI values measured with amounts of 1,500 beads/antigen/well.

Conclusion: The MBA protocol offers important advantages over ELISA for measuring antibody responses to multiple Plasmodium antigens, especially in large field studies. Reducing significantly consumables' costs and being more rapid than ELISA, the MBA protocols developed here represents a basis for standardizing assays of humoral responses and enable valid comparisons of results from different laboratories in seroepidemiological surveys or analysis of immunogenicity of vaccine candidates.

Keywords: Multiplex assay, Magnetic beads, MAGPIX technology, Malaria, Plasmodium, Antibodies detection, ELISA

\section{Introduction}

Malaria remains one of the most prevalent endemic vector-borne diseases in tropical areas worldwide. Although Plasmodium falciparum malaria is the major cause of childhood mortality and morbidity in sub-Saharan countries, four other Plasmodium species also contribute to the global burden of human malaria. The life cycle of the microscopic Plasmodium spp. pathogen causing malaria has multiple successive, antigenically distinct stages, which elicit a highly complex immune response. Antibody responses play a crucial role in protection against blood stages, but also in blocking transmission from and to the mosquito vector $[1,2]$. A protective immunity to the parasite develops in humans with increasing exposure and its acquisition is influenced by transmission intensity [3]. Protection progressively raised during childhood is maintained in adults who consequently experience asymptomatic low-density blood stage infections $[4,5]$.

In the recent years, the large-scale deployment of combined interventions strategies including insecticide impregnated bed nets, rapid diagnostic tests and efficient combination therapies, led to a remarkable decrease of malaria burden in several sub-Saharan African areas. Implementation capacity for monitoring the decrease in malaria transmission could be facilitated by measuring antibody (Ab) responses against a set of biomarkers to evaluate exposure and/or protection to malaria in populations living in endemic areas [6,7].

The breadth of antigenic make up from each malaria species parasites is such that serological correlates of exposure and protection are still uncertain. To determine antibody levels in populations living in areas where malaria is endemic, a panel of antigens (Ag) needs to be explored. As small quantities of blood are usually available, this is not feasible with the enzyme-linked immunosorbent assay (ELISA), the gold standard technique where each antigen is tested separately. A multiplexing approach is needed. Since several years, processes for simultaneous detection of antibodies to multiple antigens have been developed: multiplex assays in the format of protein microarrays [8-12] or the bead-based Luminex system [13-17] that allowed high throughput measures of multiple antibody specificities in a single assay.

One of the processes of multiplex antibody detection is based on the Luminex ${ }^{\mathrm{TM}}$ xMAP fluorescent-coded beads technology. Every single Ag can be covalently linked to a defined color-coded microsphere so that the reading device can classify each bead set separately. Prior studies using multiple malaria Ag targets have reported results with the Luminex ${ }^{100 / 200}$ instrumentation based on flow cytometry and laser detection technology. A recently available device (MAGPIX, Luminex Corporation) works with fluorescent magnetic microspheres, held in a monolayer with a magnet, illuminated by LED instead of laser system, and imaged using CCD camera, reducing cost for access to multiplex platform. To date, only few published studies provide technical information on assay optimization conditions with malaria Ags [18] and one with the use of the new MAGPIX instrument [19]. 
The present study was a pilot evaluation using a set of immune sera from individuals living in endemic areas of $P$. falciparum transmission to setup some technical constraints for a magnetic bead-based multiplex immunoassay (MBA) using the MAGPIX technology compared to standard ELISA monitoring response to each individual antigen. We firstly optimized the antigen concentration for coupling to magnetic beads using a recombinant $P$. falciparum Ag PfEMP1-PF13$\mathrm{DBL}_{1 \alpha_{1}}$ (PF13), an adhesion protein implicated in rosetting and derived from the surface of $P$. falciparum infected erythrocyte. In a second step, we tested a hexaplex combination using two recombinant antigens (PF13 and MSP1p19), three Bovine Serum Albumin (BSA)coupled synthetic peptides (CSP, $\mathrm{LSAl}_{41}$ and gSG6) and BSA as control.

We report here analysis of technical parameters that influence performance of the MBA assay and considerations for further development of this powerful technique.

\section{Material and Methods}

\section{Plasma samples}

A set of 14 serum samples from individuals living in a malaria endemic area were from adults or children living in a Senegalese village with perennial continuous transmission (Dielmo) where a long-term longitudinal survey was conducted to study acquisition and maintenance of natural immunity for more than 20 years [5,20,21]. A pool of purified Malaria Immune IgG immunoglobulin (MIG) obtained from immune African adults (a kind gift of Marcel Hommel) and a pool of plasma from adult donors living in Dielmo, Senegal (Dielmo-HIS) (distinct from the individual samples indicated above) were used as positive controls. Negative control plasmas were obtained from 25 healthy Europeans adults who had not been exposed to malaria (Blood bank, EFS-Rungis, France) and were used as a negative control pool (NIS).

\section{Antigens}

Two recombinants proteins, three BSA conjugated peptides and BSA for control was used. The PF13 Ag, derived from the P. falciparum erythrocyte membrane protein 1 (PfEMP1-NTS-DBL1a1 domain of exon 1 with a C-terminal hexa-His tag) was expressed in Escherichia coli. The coding sequence was cloned in pET21a (Novagen), and transformed into E. coli Rosetta-gami TM2 (DE3) (Novagen). Protein expression and purification have been described previously [22].

The soluble recombinant protein corresponding to $P$. falciparum merozoite surface Ag MSP1p19 corresponds to the C-terminal double EGF domain of the protein. The GPI-modification signal sequence is replaced with a hexa-histidine tag. The protein was produced in the baculovirus/High Five (Invitrogen) insect cell expression systems and purified by metalloaffinity chromatography as described previously [23].

Two $P$. falciparum peptide Ags and the gSG6 peptide derived from an A. gambiae salivary gland $\mathrm{Ag} \mathrm{[24]} \mathrm{were} \mathrm{used.}$

Two major antigens from pre-erythrocyte stages were used: $\mathrm{LSAl}_{41}$ and a repeated sequence of the circumsporozoite protein (CSP). Peptides sequences were as follows:

\section{$\mathrm{LSAl}_{41}$ :}

LAKEKLQEQQSDLEQERLAKEKLQEQQSDLEQERLAKEKEKLQCBSA;
CSP:

\section{NANPNANPNANPNANPNANPNANPNANPNANPNANPNVDPN} VDPC-BSA;

\section{gSG6: EKVWVDRDNVYCGHLDCTRVATFC-BSA.}

Peptides were synthesized with an added N-terminal cysteine residue with purity $>85 \%$ and covalently coupled with BSA (bovine serum albumin) by the manufacturer (Gen Script HK Inc., Hong Kong, China), as peptides with molecular weight smaller than $3000 \mathrm{~g} / \mathrm{mol}$ couple poorly to beads and require conjugation to BSA [13].

\section{Covalent coupling of Ag to magnetic beads}

Carboxylated Luminex magnetic beads with different fluorescence signatures (regions 12,13,14,15,18,19) (Luminex corp, Austin, USA) were covalently coupled with recombinant proteins, peptide-BSA complexes or BSA using the ready-to-use $\mathrm{xMAP}^{\circ}$ Antibody Coupling Kit (ref 40-50016, Luminex corp, Austin, USA). This one-time for use Kit contains all the reagents and suitable consumables needed to link covalently antibody or any protein to Luminex microsphere by the carbodiimide reaction. Coupling was done following manufacturers' instructions. Briefly $1.5 \times 10^{6}$ to $5 \times 10^{6}$ beads were re-suspended after sonication and rotative mixing in micro centrifuge "reaction" vials and washed twice before adding the activation buffer. The working volume was $500 \mu \mathrm{L}$; all washing steps or buffer changes were done after 1-2 min of centrifugation at $8000 \mathrm{x}$ g and $1 \mathrm{~min}$ of magnetic pelleting, followed by 30 s vortexing and sonicated $30 \mathrm{~s}$ in water-bath sonicator to optimally disperse the beads and avoid beads loss. Then, $10 \mu \mathrm{L}$ carbodiimide hypochloride (EDC) were added and incubated 20 min with an intermediate vortexing step after $10 \mathrm{~min}$. After three washes, $\mathrm{Ag}$ was added in the activation buffer for a final volume of $500 \mu \mathrm{L}$ and kept under rotation mixing (15-30 rpm) in the dark for 2 hours. After three steps of pelleting and washing, the supernatant was removed and replaced by $1 \mathrm{~mL}$ washing buffer and kept in the dark at $2-8^{\circ} \mathrm{C}$ after another vortexing and sonication step to disperse the microspheres. Final count of remaining beads was done using cell counter, the coupled microspheres were kept in the washing/storage buffer at $4^{\circ} \mathrm{C}$ in the dark until further use.

\section{Monoclonal antibodies}

To confirm the bead coupling specificity and efficiency of PF13 and PfMSP1-p19 soluble recombinant proteins, mouse monoclonal antibodies (mAb) directed against PF13 (mAbs J3.21), or PfMSP1-p19 (mAb G17.12, kind gift from Dr Shirley Longacre). These $\mathrm{mAb}$ were used instead of sera at different dilutions.

\section{Multiplex magnetic bead-based immunoassay (MBA)}

The procedure was performed in a dimly lit room. After counting Ag-coated bead, an equal number of beads for each fluorescent bead region was mixed and distributed in a 96-well white polystyrene round bottom micro titer plate (Corning, Ref 3789, Fisher Scientific) to reach a final concentration of 1500 beads/region/Ag in each well. The plate was subsequently placed on a Luminex Magnetic Plate Separator (Luminex, ref CN-0269-01) to remove the supernatant and $100 \mu \mathrm{L}$ plasma/serum at the appropriate dilution were added in duplicate wells. Plasma or serum dilutions were done in PBS 1x supplemented with $0.05 \%$ Tween 20 and $1 \%$ of BSA (PTB buffer). Plates, protected from light, were incubated at room temperature $\left(18-22^{\circ} \mathrm{C}\right)$ for $45 \mathrm{~min}$ under constant shaking $(350 \mathrm{rpm})$. Following this incubation step, plates were washed three times in PTB buffer and $100 \mu \mathrm{L}$ secondary 
antibody (R-phycoerythrin conjugated goat $\mathrm{F}(\mathrm{ab}$ ') 2 anti-humans IgG, Molecular Probes/Life Technologies, H10104) diluted at 1/500 in PTB buffer was added in each well. After 45 min incubation in the dark at room temperature $\left(18-22^{\circ} \mathrm{C}\right)$ under constant shaking $(350 \mathrm{rpm})$, plates were carefully washed twice with PTB buffer. Beads were finally resuspended in $120 \mu \mathrm{L}$ PTB and plates were analyzed using the MAGPIX ${ }^{\circ}$ Luminex system (Millipore, USA) and the xPONENT 4.1 software for acquisition and assay design. The reader was set to read a minimum of 50 beads per spectral address and the output measured as Median Fluorescence Intensity (MFI) as stated by manufacturer's instruction of use. The level of antibody responses was expressed in MFI, positive responders were individuals with MFI signal over the mean MFI level of naive controls $+2 \mathrm{SD}$.

\section{Enzyme-Linked Immunosorbent Assay (ELISA)}

ELISA assays were performed using plasma samples diluted 1:200. The procedure used to measure seroprevalence and levels of antibodies against PF13, PfMSP1p19 and peptides has been reported [19,25,26]. Recombinant antigens and BSA-peptides were coated on Immulon-4 plates (Dynatech) at $1 \mu \mathrm{g} \cdot \mathrm{mL}^{-1}$ and BSA (provided from peptide manufacturer) was coated at $0.5 \mu \mathrm{g} / \mathrm{mL}^{-1}$. Each plate included positive controls, namely MIG and HIS and a negative naive control (Pool of non-Immune European sera). For reliable inter-assay comparisons, results were expressed as OD-ratios corresponding to OD-sample/ODnaive. Positive responders were individuals with an OD-ratio over 2, corresponding to the mean OD of naïve controls $+2 \mathrm{SD}$.

\section{Statistical Analysis}

The antibody responses were not normally distributed (Shapiro test) and hence, non-parametric tests were used for analyses. Continuous variables OD ratios and MFI were compared using the Spearman rank for analysis of correlation. Correlation was considered significant for $\mathrm{P}$ values $<0.05$. Statistical analyses were performed with Stat view $5.0^{\circ}$ software.

\section{Results}

\section{Optimization of Ag coupling conditions}

The covalent coupling of Ag to color-coded magnetic beads was initially realized following manufacturers' instructions. However, as shown in Table 1, bead recovery was variable and ranged from $62 \%$ to $100 \%$. Bead loss was unrelated to the $\mathrm{Ag}$ (recombinant protein or peptide-BSA) used and was observed even if $2.5 \times 10^{6}$ magnetic beads were used. To avoid excessive loss of magnetic beads during the coupling procedure, a centrifugation step was added before magnetic bead separation to ensure efficient pelleting (see Materials and Methods section).

To determine the optimal Ag amount to be used for bead coupling, three concentrations of PF13 recombinant protein (1, 10 and $20 \mu \mathrm{g}$ per $10^{6}$ beads) were tested using a single vial of "low" concentration region of beads i.e. $2.5 \times 10^{6}$ beads $/ 3=0.83 \times 10^{6}$ beads per assay. When 10 or $20 \mu \mathrm{g}$ protein per $10^{6}$ beads was used, a large amount of uncoupled $\mathrm{Ag}$ was revealed in the post-conjugation supernatant as detected by ELISA using mAb J3.21 (Figure 1). To check that the presence of unbound protein in the supernatant was not due to ineffective coupling, PF13coupled beads were tested in monoplex MBA using the positive control pools (MIG and HIS), the negative control pool (NIS) and serial dilutions of an anti-PF13 mouse polyclonal serum. As illustrated in Figure 2, similar levels of MFI were observed between the 3 concentrations of PF13 protein tested when human (left panel) or mouse antibodies (right panel) were used.

\begin{tabular}{|l|l|l|l|l|l|}
\hline \multicolumn{1}{|c|}{ Antigen } & \multicolumn{2}{c|}{ Magnetic Beads } \\
\hline Identification & \multicolumn{1}{|c|}{ Quantity } & Region ID & Recovery \\
\hline PF13 & $1 \mu \mathrm{g} / 10^{6}$ & 13 & $0.8310^{6}$ & $0.7310^{6}$ & 88 \\
\hline PF13 & $10 \mu \mathrm{g} / 10^{6}$ & 13 & $0.8310^{6}$ & $0.6410^{6}$ & 77 \\
\hline MSP1p19 & $20 \mu \mathrm{g} / 10^{6}$ & 13 & $0.8310^{6}$ & $2.7910^{6}$ & $10^{6}$ \\
\hline PF13 & $5 \mu \mathrm{g} / 10^{6}$ & 12 & $2.510^{6}$ & $2.5010^{6}$ & 95 \\
\hline CSP & $5 \mu \mathrm{g} / 10^{6}$ & 13 & $2.510^{6}$ & $1.5510^{6}$ & 100 \\
\hline LSA141 & $5 \mu \mathrm{g} / 10^{6}$ & 14 & $2.510^{6}$ & $1.9510^{6}$ & 72 \\
\hline gSG6 & $5 \mu \mathrm{g} / 10^{6}$ & 15 & $2.510^{6}$ & $2.3010^{6}$ \\
\hline BSA & $5 \mu \mathrm{g} / 10^{6}$ & 18 & $2.510^{6}$ & $2.4010^{6}$ \\
\hline
\end{tabular}

Table 1: Summary of the final magnetic beads recovery after the coupling procedures for the different antigen tested 


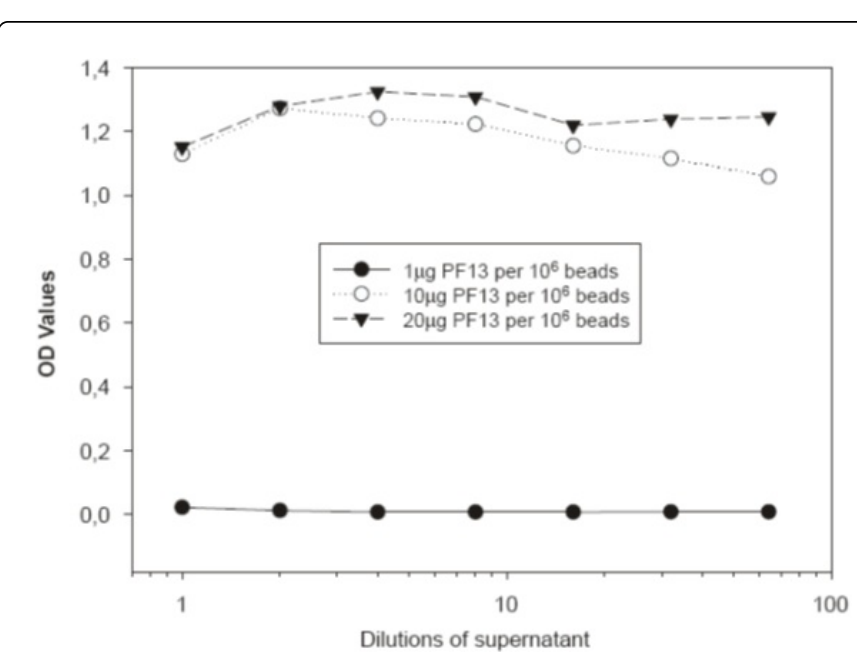

Figure 1: Detection of PF13 Ag in supernatants after coupling procedure using different concentration of the protein Increasing dilutions of supernatants after the first coupling procedure for PF13 were coated overnight at $4^{\circ} \mathrm{C}$ on Imulon IV ELISA plates. The presence of PF13 was revealed by a polyclonal anti-PF13 mouse antibody diluted 1:5000 and plotted as OD values on $\mathrm{Y}$ axis, showing a detectable excess of unbound Ag over $1 \mu \mathrm{g}$ per 106 beads.

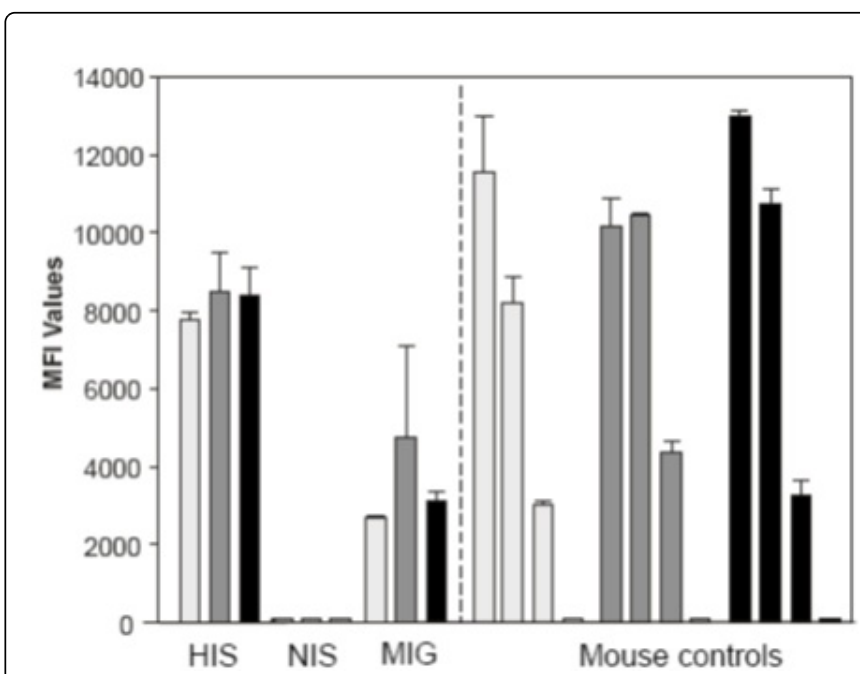

Figure 2: MFI values of human and mouse control sera IgG antibody levels against PF13 coupled at different concentrations to the fluorescent magnetic beads MFI values measured using the different Ag-coupled beads are shown for: (i) the immune HIS pool, (ii) the NIS negative pool, (iii) the immune MIG pool, (iv) mouse anti-PF13 polyclonal serum diluted 1:1,000, 1:5,000, 1:10,000 (from left to right) and (v) mouse pre-immune control diluted 1:1,000, $1: 5,000,1: 10,000$ (from left to right). Beads coupled with 1, 10 and $20 \mu \mathrm{g}$ per 106 beads are shown in pale grey, dark grey and black, respectively. A dose-effect output was evidenced with the mouse sera.

\section{Specificity of the MBA test}

The monoplex with PF13-beads coupled with the three protein concentrations used above (Figure 2) showed an excellent specificity of the technique, with a good dynamic range with the positive control pools and negligible signals with negative control NIS pool. The positive signal was high whatever the initial concentration of $\mathrm{Ag}$ used for cross-linking. The excellent background to noise ratio was confirmed using the specific mouse anti-PF13 serum. The semiquantitation performed with the mouse anti-PF13 serum showed higher signals at dilution 1:5,000 with the beads coupled with 10 or 20 $\mu \mathrm{g}$. More than two steps of washing or longer incubation steps (1 hour) did not change background and positive signals. These results led us choose an intermediate concentration of $5 \mu \mathrm{g}$ recombinant protein or peptide-BSA per $10^{6}$ beads for the subsequent couplings, use for 45 min-long incubation steps and perform two steps of washing.

\section{Concordance between MBA and ELISA}

Optical density (OD) values measured by ELISA and MFI values obtained MBA were compared for 14 plasma samples from Dielmo, a rural Senegalese village where malaria transmission was intense and perennial at the time of the sampling. As shown in Table 2, correlation between the two techniques, calculated by Spearman rank test, was strong (rho from 0.50 to 0.86$)$ and highly significant $(\mathrm{P}<0.01)$ for all antigens, including BSA $(\mathrm{P}=0.03)$. These $\mathrm{Ag}$ were differently recognized by individuals, Figure 3 details individual results expressed as a 3-level stratified antibody responses namely negative vs. intermediate vs. strong (i.e. larger than mean values) showing interindividual variation and concordance between the two methods.

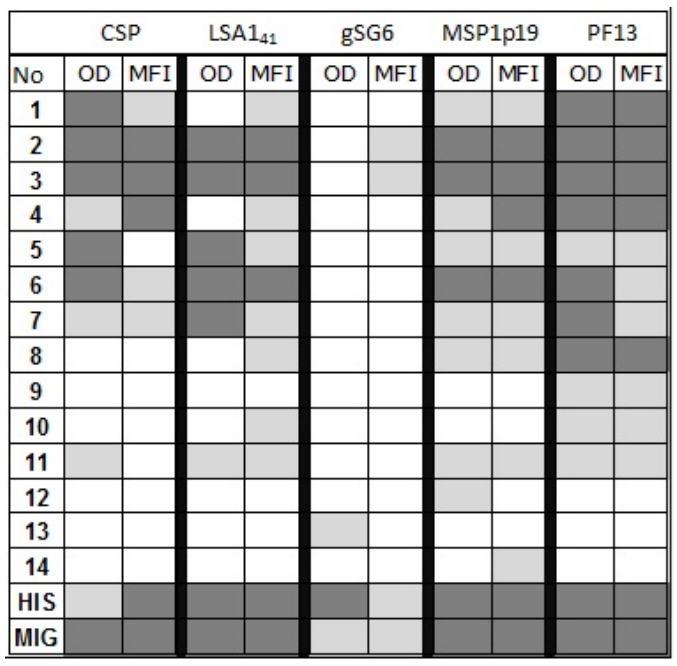

Figure 3: Comparison of ELISA vs. MBA output for individual sera Antibody responses against the 5 antigens are detailed for individual sera and controls. It has been expressed as a 3-level stratified responses namely negative vs. positive (OD ratio [OD] or $\mathrm{MFI}>$ naïve background+2SD) vs. strong (i.e. OD ratio or MFI > mean value of the set of sera tested). Inter-individual variations and general concordance between the two methods are underlined. HIS=Human Immune Serum; MIG=Malaria Immune Globulins are the positive controls 


\begin{tabular}{|l|l|l|l|l|l|l|}
\hline & BSA & CSP Ag & LSA1 ${ }_{41}$ Ag & gSG6Ag & MSP1p19 & PF13 Ag \\
\hline OD values $^{*}$ & $0.16[0.02-0.85]$ & $0.27[0.00-0.73]$ & $0.37[0.01-2.09]$ & $\begin{array}{l}0.07 \\
{[0.00-0.57]}\end{array}$ & $1.01[0.04-2.70]$ & $1.39[0.04-2.28]$ \\
\hline Odratio $^{*}$ & $3.7[1-17.1]$ & $6.3[1-14.4]$ & $6.7[1-20.3]$ & $1.0[1-2.6]$ & $13.6[1-43.1]$ & $18.8[1-36.4]$ \\
\hline MFI values $^{*}$ & $35[12-170]$ & $107[14-733]$ & $865[16-4487]$ & $\begin{array}{l}138 \\
{[16-930]}\end{array}$ & $306[21-1283]$ & $1052[27-3373]$ \\
\hline correlation $^{* *}$ & 0.50 & 0.61 & 0.86 & 0.73 & 0.77 & 0.82 \\
\hline $95 \% \mathrm{Cl}^{\circ}$ & $0.03-0.79$ & $0.18-0.84$ & $0.65-0.95$ & $0.39-0.89$ & $0.46-0.91$ & $0.56-0.93$ \\
\hline $\mathrm{P}^{\circ}$ & 0.03 & 0.007 & $<10-4$ & $<10-3$ & $<10-3$ & $<10-4$ \\
\hline
\end{tabular}

Table 2: Summary of the levels of responses and correlation coefficients between ELISA and MBA assays for the different antigen tested

\section{Evaluation of serial plasma dilution, bead count and reproducibility over time}

The degree of association between MFI and OD values was tested with a serial dilution of the two positive HIS MIG controls diluted 1:100 to 1:10,000. Results are plotted on Figure 4, showing high titers of antibodies to PF13, MSP1p19 and LSA1 ${ }_{41}$, slightly lower titers to CSP (around 1:2,000) and very low levels of antibody to the $A$. gambiae salivary peptide antigen.
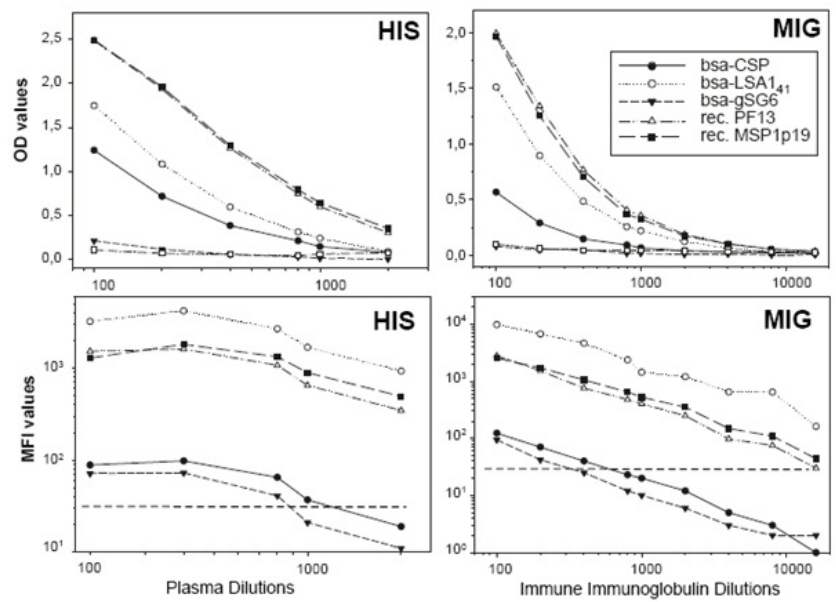

Figure 4: OD values and MFI values from serial dilutions of positive controls i.e. pool of immune sera (HIS) and immune IgG (MIG) $\mathrm{OD}$ values and MFI values of $\mathrm{IgG}$ responses to the 5 different $\mathrm{Ag}$ coupled beads are plotted for serial dilutions of the positive controls: HIS and MIG. MFI values were plotted as $\log _{10}$ transformed signal. The use of the two methods led to similar profiles for antibody level evaluation.

We found similar results and profiles of antibody titration by the two methods and the background signal against BSA was negligible. However, there was a slight difference between OD and MFI measures regarding anti-LSA1 $1_{41}$ antibody content with a higher level of MFI signal then OD values, when compared to PF13 and MSP1p19.

The validation of multiplex antibody detection MFI measures requires an optimal number of beads. In the MAGPIX system with
$\mathrm{xPonent}$ software, the minimum manufacture's requirement is 50 beads per region for statistical validation of MFI calculation (indicated as a green toggle in each well on the screen output).

When the number of events was increased (100 or 150 beads per region), the MFI results in the same assay did not significantly change. Thus, the recommended minimum setting of 50 beads count per region per well allows to run a second set of counts, if necessary for further checking, with the same plate on the next day after keeping the plates at $5-8^{\circ} \mathrm{C}$ in the dark. Results obtained did not significantly differ from the first measure ( $<5 \%$ variation).

Regarding bead stability over time, the same hexaplex mix kept during 4 months at $4^{\circ} \mathrm{C}$ has been tested; it included the two positive reference controls and the set of 14 sera. We found similar results in MFI with some variations in the individual MFI data (from $8 \%$ to $150 \%)$. The correlation between results of the two sets of measures was highly significant $(\mathrm{Rho}=0.93, \mathrm{P}<10-3)$. Variations of large amplitude were from negative or very low responders; individual plasma with high initial MFI levels showed $<15 \%$ of variation after 4 -month conservation.

\section{Discussion}

The multiplex fluorescent bead assay is a powerful technique usually used with instrumentation based on flow cytometry and laser detection technology. Several custom approaches have been published, and numerous commercial kits are available based on Ag capturerelated assay by monoclonal antibodies. An increasing number of kits are now available prepared with magnetic beads coupled to specific immune reagents or Ags. Multiplex assays have a large panel of applications such as determination of cytokines, viral markers, bacterial markers for water or food testing, multi-antigen detection for transplantation, cancer immunology etc. [27-30]

In the case of malaria parasites, custom-ELISA multiplex analyses of selected Ags have been recently published. The antibody responses are monitored using of a variety of protocols [13,16,24,32]. Some investigations provide information on assay optimization conditions with the Luminex ${ }^{100}$ system [18].

In this work, we wanted to explore the antibody response to antigens that have been explored in previous studies using ELISA $[19,26]$ and shown to have a high seroprevalence in this Senegalese setting. The final aim is to perform a longitudinal analysis of the antibody response of the villagers during the last decade and use for 
future studies a multiplexing technological approach allowing meaningful comparisons with previous serological surveys. In the work reported here, we set several technical points that should be taken into account for further development with additional antigens.

A first obstacle was an excessive loss of beads during the coupling procedure and when managing the MBA test. For the coupling procedure itself, the use of a commercial kit was a comfortable solution providing adapted "non-sticky" plastic materials. We introduced a double pelleting of the beads at each step, both by centrifugation and use of the magnet. The second parameter is the production of the multiplex reagent; it is a major step for mounting the custom multiplex assay requiring attention. The quality of the recombinant proteins or BSA-peptide conjugates and the buffer in which they are presented to the beads for carbodiimide crosslinking are important factors. The commercial kit allowed preparing a multiplex test with more than 10 antigens at the same time, with a minimum loss of beads. When performing the tests with plasma, a substantial loss of beads was sometimes observed, this precluded MFI measurement. This problem was solved by using round bottom micro titer plates and by limiting the number and the duration of washing steps. Two washing steps were sufficient (contrary to ELISA), as evidenced by the negligible background MFI signal for the negative controls.

We also explored the amount of $\mathrm{Ag}$ and beads to be used in MBA. Coupling of 5ug of antigen per $10^{6}$ beads (recombinant protein or BSA-coupled peptide) was sufficient to saturate the beads. In published reports, concentrations of antigen evaluated ranged from 0.5 to $100 \mathrm{ug}$ per $10^{6}$ beads, depending upon the antigen used. An amount of 1 to 5 ug per $10^{6}$ beads was usually found optimal $[15,16,32,33]$, except for bacterial crude extract requiring a higher amount of protein [28]. We finally selected 5ug of $\mathrm{Ag}$ for coupling: it was an intermediate value between the two amount of PF13 tested here $(1 \mu \mathrm{g}$ and $10 \mu \mathrm{g})$, and seems over sufficient for coupling with minimum loss of Ag.

In order to measure of antibody levels, 3000-5000 beads per antigen per well is the usually recommended range. In the MAGPIX system, the capacity measurement is overloaded when introducing 60,000 beads per well (manufacturer's information) i.e. a maximum of 12 Ags (5000 beads per Ag per well, 0\% loss during the procedure). To set up an assay with a higher number of Ags (which is our final goal), optimization of the test using the lowest amount possible of antigencoupled beads was required. We used here the usual load of 3000 beads per Ag per well. In addition, combining careful pelleting with a minimum number of washing steps allowed us to lower the input to 1500-2000 beads, leading to $100 \%$ of wells with multiplexed MFI data in each plate. And indeed, subsequent assays using 16 different peptides or recombinant antigens resulted in easily interpretable individual data, with no cross reactivity and an excellent signal to noise ratio (data not shown).

An important point is the presentation of the antigen itself using this technique. The beads in the assay anchor the antigen by covalent linking of a carbodiimide linker to Lysine residues of the protein, as opposed to ELISA where the coating of surfaces of the wells by the antigen results from non-specific hydrophobic interactions with the plastic plate. As a consequence, it is likely that surface epitopes are not presented in exactly the same configuration and binding efficiency and fine specificity of antibody, therefore measures may differ when using these two techniques. Thus it is important to compare the performance of both assays and cross validate results. Prior studies using multiplex assay for antibody determination to $P$. falciparum antigens checked for concordance with ELISA monoplex $[13,15]$. In agreement with the observations reported here, there was a high degree of correlation between both methodologies [15,33,34]. We observed such a high correlation in a recently published study [19] that used the optimized MBA protocol reported here. However, some variations can be observed at the individual level.

The dynamic range of MFI values (up to 200-fold over background levels) is larger than the OD range of the ELISA (30-60-fold the background signal). This may account for the different shape of titration curves obtained with the two techniques on Figure 3, which contrast with an early report with a $P$. falciparum bead assay [15]. Alternatively, as indicated above, this may reflect the partial overlap of the set of epitopes presented for antibody binding in the two methodologies.

Another major advantage of multiplex assays is their versatility: biomarkers from different Plasmodium species can be investigated to detect $P$. falciparum as well as $P$. vivax or P malariae or P. knowlesi in areas where these species are endemic. Moreover, multiplexing allows screening a large panel of antigens to be used as biomarkers and generate antigen combinations with the best value as biomarkers of infection.

\section{Conclusion}

The aim of the present study was to setup and share some technical basis for a MAGPIX -based approach compared to ELISA. We developed MBA so as to use a procedure similar to the ELISA usual procedure, using the same diluents and serum dilutions and sharing the same standards. We optimized the assay to minimize loss of beads at the successive steps from Ag coupling to incubation and washings of the plates. The method described here provides a platform to further develop the MBA methodology, using additional peptides or recombinant antigens and reference reagents.

We think that this affordable multiplex methodology (approx. 0.5 USD per individual per antigen) is amenable to widespread use in endemic settings and offers interesting opportunities to evaluate and validate panels of Ag as biomarkers in seroepidemiology studies.

\section{Acknowledgements}

We are grateful to the villagers of Dielmo for their active participation and continuing collaboration in the project. We thank Dr $\mathrm{S}$ Longacre for constant support and Ag providing. We thank Drs M Jouan and J Salomon from Division International (Institut Pasteur, Paris) for their help to initiate this work in the very early steps.

\section{Financial support}

This study was supported by grants from Institut Pasteur, from Rotary International and from ACIP (Institut Pasteur, N²5_2012)

\section{References}

1. Bouharoun-Tayoun $\mathrm{H}$, Attanath $\mathrm{P}$, Sabchareon A, Chongsuphajaisiddhi T, Druilhe P (1990) Antibodies that protect humans against Plasmodium falciparum blood stages do not on their own inhibit parasite growth and invasion in vitro, but act in cooperation with monocytes. J Exp Med 172: 1633-1641.

2. Cohen S, Mcgregor IA, Carrington S (1961) Gamma-globulin and acquired immunity to human malaria. Nature 192: 733-737.

3. WHO (2013) World Malaria Report. 
4. Doolan DL, Dobaño C, Baird JK (2009) Acquired immunity to malaria. Clin Microbiol Rev 22: 13-36, Table of Contents.

5. Trape JF, Rogier C (1996) Combating malaria morbidity and mortality by reducing transmission. Parasitol Today 12: 236-240.

6. Bousema T, Okell L, Felger I, Drakeley C (2014) Asymptomatic malaria infections: detectability, transmissibility and public health relevance. Nat Rev Microbiol 12: 833-840.

7. Bretscher MT, Supargiyono S, Wijayanti MA, Nugraheni D, Widyastuti AN, et al. (2013) Measurement of Plasmodium falciparum transmission intensity using serological cohort data from Indonesian schoolchildren. Malar J 12: 21.

8. Gray JC, Corran PH, Mangia E, Gaunt MW, Li Q, et al. (2007). Profiling the antibody immune response against blood stage malaria vaccine candidates. Clin Chem 53: 1244-1253.

9. Parekh FK, Richie TL (2007) Characterization of immune reactivity profiles using microarray technology may expedite identification of candidate antigens for next generation malaria vaccines. Clin Chem 53: 1183-1185.

10. Baum, E., Badu, K., Molina, D.M., Liang, X., Felgner, P.L., et al. (2013). Protein microarray analysis of antibody responses to Plasmodium falciparum in western Kenyan highland sites with differing transmission levels. PLoS One 8, e82246.

11. Crompton PD, Kayala MA, Traore B, Kayentao K, Ongoiba A, et al. (2010) A prospective analysis of the $\mathrm{Ab}$ response to Plasmodium falciparum before and after a malaria season by protein microarray. Proc Natl Acad Sci U S A 107: 6958-6963.

12. Crosnier C, Wanaguru M, McDade B, Osier FH, Marsh K, et al. (2013) A library of functional recombinant cell-surface and secreted P. falciparum merozoite proteins. Mol Cell Proteomics 12: 3976-3986.

13. Ambrosino E, Dumoulin C, Orlandi-Pradines E, Remoue F, Toure-Balde A, et al. (2010) A multiplex assay for the simultaneous detection of antibodies against 15 Plasmodium falciparum and Anopheles gambiae saliva antigens. Malar J 9: 317.

14. Cham GK, Kurtis J, Lusingu J, Theander TG, Jensen AT, et al. (2008). A semi-automated multiplex high-throughput assay for measuring IgG antibodies against Plasmodium falciparum erythrocyte membrane protein 1 (PfEMP1) domains in small volumes of plasma. Malar J 7: 108.

15. Fouda GG, Leke RF, Long C, Druilhe P, Zhou A, et al. (2006) Multiplex assay for simultaneous measurement of antibodies to multiple Plasmodium falciparum antigens. Clin Vaccine Immunol 13: 1307-1313.

16. Dodoo D, Atuguba F, Bosomprah S, Ansah NA, Ansah Ps, et al. (2011). Antibody levels to multiple malaria vaccine candidate antigens in relation to clinical malaria episodes in children in the Kasena-Nankana district of Northern Ghana. Malar J 10: 108.

17. Proietti C, Verra F, Bretscher MT, Stone W, Kanoi BN, et al. (2013) Influence of infection on malaria-specific antibody dynamics in a cohort exposed to intense malaria transmission in northern Uganda. Parasite Immunol 35: 164-173

18. Ondigo BN, Park GS, Gose SO, Ho BM, Ochola LA, et al. (2012) Standardization and validation of a cytometric bead assay to assess antibodies to multiple Plasmodium falciparum recombinant antigens. Malar J 11: 427.

19. Perraut R, Richard V, Varela ML, Trape JF, Guillotte M, et al. (2014) Comparative analysis of IgG responses to Plasmodium falciparum MSP1p19 and PF13-DBL1alpha1 using ELISA and a magnetic bead-based duplex assay (MAGPIX ${ }^{\oplus}(\mathrm{R})$-Luminex) in a Senegalese meso-endemic community. Malar J 13: 410.

20. Garraud O, Mahanty S, Perraut R (2003) Malaria-specific antibody subclasses in immune individuals: a key source of information for vaccine design. Trends Immunol 24: 30-35.

21. Trape JF, Rogier C, Konate L, Diagne N, Bouganali H, et al. (1994) The Dielmo project: a longitudinal study of natural malaria infection and the mechanisms of protective immunity in a community living in a holoendemic area of Senegal. Am J Trop Med Hyg 51: 123-137.

22. Vigan-Womas I, Guillotte M, Juillerat A, Vallieres C, LewitBentley A, et al. (2011) Allelic diversity of the Plasmodium falciparum erythrocyte membrane protein 1 entails variantspecific red cell surface epitopes. PLoS One 6: e16544.

23. Bonnet S, Petres S, Holm I, Fontaine T, Rosario S, et al. (2006) Soluble and glyco-lipid modified baculovirus Plasmodium falciparum C-terminal merozoite surface protein 1, two forms of a leading malaria vaccine candidate. Vaccine 24: 5997-6008.

24. Rizzo C, Ronca R, Fiorentino G, Verra F, Mangano V, et al. (2011) Humoral response to the Anopheles gambiae salivary protein gSG6: a serological indicator of exposure to Afrotropical malaria vectors. PLoS One 6: e17980.

25. Perraut R, Marrama L, Diouf B, Fontenille D, Tall A, et al. (2003) Distinct surrogate markers for protection against Plasmodium falciparum infection and clinical malaria identified in a Senegalese community after radical drug cure. J Infect Dis 188: 1940-1950.

26. Vigan-Womas I, Guillotte M, Le Scanf C, Igonet S, Petres S, et al. (2008) An in vivo and in vitro model of Plasmodium falciparum rosetting and autoagglutination mediated by varO, a group A var gene encoding a frequent serotype. Infect Immun 76: 5565-5580.

27. Elgueta S, Fuentes C, López M, Hernández J, Arenas A, et al. (2011) Effect of implementing anti-HLA antibody detection by Luminex in the kidney transplant program in Chile. Transplant Proc 43: 3324-3326.

28. Griffin SM, Chen IM, Fout GS, Wade TJ, Egorov AI (2011) Development of a multiplex microsphere immunoassay for the quantitation of salivary antibody responses to selected waterborne pathogens. J Immunol Methods 364: 83-93.

29. Moncunill G, Mayor A, Jimenez A, Nhabomba A, Puyol L, et al. (2013) Cytokine and Antibody Responses to Plasmodium falciparum in Naive Individuals during a First Malaria Episode: Effect of Age and Malaria Exposure. PLoS One 8: e55756.

30. Moss DM, Priest JW, Boyd A, Weinkopff T, Kucerova Z, et al. (2011) Multiplex bead assay for serum samples from children in Haiti enrolled in a drug study for the treatment of lymphatic filariasis. Am J Trop Med Hyg 85: 229-237.

31. Riley EM, Stewart VA (2013) Immune mechanisms in malaria: new insights in vaccine development. Nat Med 19: 168-178.

32. Sarr JB, Orlandi-Pradines E, Fortin S, Sow C, Cornelie S, et al. (2011) Assessment of exposure to Plasmodium falciparum transmission in a low endemicity area by using multiplex fluorescent microsphere-based serological assays. Parasit Vectors 4: 212 .

33. Fernandez-Becerra C, Sanz S, Brucet M, Stanisic DI, Alves FP, et al. (2010) Naturally-acquired humoral immune responses against the N- and C-termini of the Plasmodium vivax MSP1 protein in endemic regions of Brazil and Papua New Guinea using a multiplex assay. Malar J 9: 29. 


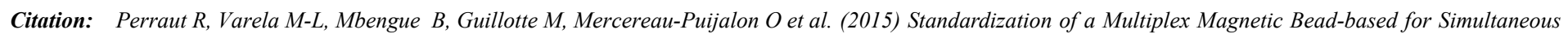
Detection of IgG to Plasmodium Antigens. J Immunol Tech Infect Dis 4:2.

34. Cham GK, Kurtis J, Lusingu J, Theander TG, Jensen AT, et al. (2008) A semi-automated multiplex high-throughput assay for measuring IgG antibodies against Plasmodium falciparum erythrocyte membrane protein 1 (PfEMP1) domains in small volumes of plasma. Malar J 7: 108. 